\title{
RESONANT GREEN'S FUNCTION FOR EULER-BERNOULLI BEAMS BY MEANS OF THE FREDHOLM ALTERNATIVE THEOREM
}

\author{
Seyed Mojtaba Hozhabrossadati ${ }^{1}$, Ahmad Aftabi Sani ${ }^{2}$, Masood Mofid ${ }^{3}$ \\ ${ }^{1,2}$ Department of Civil Engineering, Mashhad Branch, Islamic Azad University, Mashhad, Iran \\ ${ }^{3}$ Department of Civil Engineering, Sharif University of Technology, Tehran, Iran \\ ${ }^{1}$ sm.hozhabrossadati@yahoo.com, 2 aftabi.ahmad@gmail.com, ${ }^{3}$ mofid@sharif.edu
}

\begin{abstract}
This paper presents the Green's function for a uniform thin beam which is assumed to obey the Euler-Bernoulli theory at resonant condition. The beam under study has a simple support at one end and a sliding support at the other. First, the differential equation governing the free vibration of the beam is obtained in the frequency domain using the Fourier transform. Then, we try to find the corresponding Green's function of the problem. But a contradiction occurs due to the special properties of resonance. In order to overcome this hurdle, the Fredholm Alternative Theorem is utilized. Remarkably, it is shown that this theorem, by adding a particular term to the Green's function, can remedy this problem and the modified Green's function is consequently established. Moreover, the deformation function of the beam is found in an integral equation form. Some diagrams and tables conclude this study.
\end{abstract}

Keywords: Fredholm Alternative Theorem, modified Green's function, resonance, Euler-Bernoulli beam

\section{Introduction}

Continuous structural systems are encountered in many branches of engineering, such as civil, ocean, aerospace and mechanical engineering. Thus, enormous importance is attached to the dynamic analysis of such systems. To this end, an effective method is the Green's function method which has been extensively utilized by researchers for the solution of vibrating beams. Xu and Cheng [1] used the Green's function method in vibration of beams with intermediate elastic supports and elastically mounted concentrated masses. They applied Green's functions to change the governing equation of the system into an integral equation. Moreover, they derived the frequency equation in terms of the standard eigenvalue problem of a matrix and calculated it numerically. Green's functions for beams of several common boundary conditions, as well as their relevant derivatives have been tabulated by Mohamad [2]. In addition, natural frequencies and mode shapes of beams with intermediate attachments were presented in his work. Kukla [3] considered the use of Green's function in the frequency analysis of a combined system consisting 
of a Timoshenko beam and multi-mass oscillators. He showed that in the case of the beam with a spring-mass system attached, the frequencies of the system lower than the spring-mass frequency are decreased, and the higher ones are increased (except at discrete points when the frequencies are unchanged).

Soon after, Foda and Abduljabbar [4] presented an exact and direct modeling technique based on the dynamic Green's function for the modeling beam structures subjected to a mass moving at constant speed. The equation of motion in the matrix form was formulated and was non-dimensionalized, so that the numerical results presented are applicable to large combinations of system parameters. A method of determining the dynamic response of uniform damped Euler-Bernoulli beams subjected to distributed and concentrated loads was presented by Abu-Hilal [5]. Also Green's function for various beams with different boundary conditions were given.

Moreover, the use of the static Green's function in engineering mechanics is common. For instance, Failla and Santini [6] revealed that uniform-beam static Green's functions can be used to build efficient solutions for beams with internal discontinuities due to along-axis constraints and flexural-stiffness jump. The equilibrium equation was derived in the space of generalized functions, and the original bending problem was recast as linear superposition of a principal and an auxiliary bending problem, both involving a uniform reference beam and homogeneous boundary conditions. Later on, Failla [7] presented a solution using static Green's functions for Euler-Bernoulli arbitrary discontinuous beams acted upon by arbitrary static loads. For stepped beams with internal springs, a closed-form solution was proposed, while for stepped beams with internal springs and along-axis supports, the solution was given in terms of the unknown reactions of the along-axis supports, which could be computed based on pertinent conditions. It is worth mentioning that Azizi et al. [8] used the spectral element method in frequency domain to analyze continuous beams and bridges subjected to a moving load. They utilized the static Green's function as a modifying function to improve the moment and shear force results. As it is mentioned, the Green's function method is extensively treated by researchers. However, no published manuscript has heretofore covered the application of this method in the dynamic analysis of structures in the presence of resonance. This paper attempts to fill this void in the world of texts.

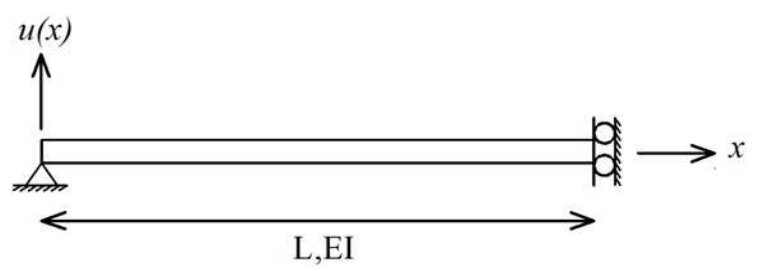

Fig. 1. The beam under study

In this paper, dynamic analysis of an Euler-Bernoulli beam with one simple and one sliding support as shown in Figure 1 in the presence of resonance is investigated, using Green's function method. The analysis is carried out in the frequency 
domain by means of the well-known Fourier transform. The paper is started by establishing the governing differential equation of Euler-Bernoulli beams in the frequency domain. The Green's function of the studied beam at the resonant condition is then to be constructed which a contradiction occurs, due to the special situation of the resonance. It is shown that the construction of the Green's function of the problem requires a new theorem to be introduced: the Fredholm Alternative Theorem. Thus, the modified Green's function of the problem is found and the above-mentioned contradiction will be eliminated. As a result, the resonant response of the beam will be achieved. In the next section, some numerical examples and discussion concludes the paper.

\section{Problem formulation}

The governing differential equation of the transverse vibration of a uniform thin beam employing the Euler-Bernoulli theory can be expressed as [9]

$$
\mathrm{EI} \frac{\partial^{4} U(x, t)}{\partial x^{4}}+\mathrm{m} \frac{\partial^{2} U(x, t)}{\partial t^{2}}=Q(x, t)
$$

where $U(x, t)$ and $Q(x, t)$ are the functions of transverse displacement and transverse loading, and EI and $\mathrm{m}$ are two constants which denote the flexural stiffness and the mass per unit length of the beam, respectively.

A well-known method for the solution of PDEs is the use of the Fourier transform. Thus, applying the Fourier transform with respect to $t$ to Eq. (1) and dividing both sides by EI gives

$$
u^{\prime \prime \prime \prime}-\lambda^{4} u=\phi(x)
$$

in which $u$ and $\phi$ are the products of the Fourier transform of $U$ and $Q$, respectively. Moreover, $\lambda=\mathrm{m} \omega^{2} / \mathrm{EI}$ is the frequency parameter of the beam. Eq. (3) could be considered as the governing equation of the Euler-Bernoulli beam in the frequency domain. In the next section, we attempt to solve the aforesaid differential equation, utilizing the Green's function method.

\section{Green's function}

The Green's function of the problem is the solution of the following equation

$$
G^{\prime \prime \prime \prime}(x, \xi)-\lambda^{4} G^{\prime \prime \prime \prime}(x, \xi)=\delta(x-\xi)
$$

or

$$
\mathbf{L}^{*} G(x, \xi)=\delta(x-\xi)
$$


where $\mathbf{L}^{*}$ is the adjoint operator of the differential operator of the problem $\mathbf{L}$. It can be readily shown that $\mathbf{L}$ is self-adjoint, i.e., $\mathbf{L}=\mathbf{L}^{*}$.

The solution of Eq. (4) is assumed

$$
G(x, \xi)= \begin{cases}a \sin (\lambda \xi)+b \cos (\lambda \xi)+c \sinh (\lambda \xi)+d \cosh (\lambda \xi) & 0 \leq \xi \leq x \\ e \sin (\lambda \xi)+f \cos (\lambda \xi)+g \sinh (\lambda \xi)+h \cosh (\lambda \xi) & x \leq \xi \leq L\end{cases}
$$

in which $a-h$ are constants and are evaluated such that the Green's function satisfies the following conditions [4]:

(a) two boundary conditions at each end of the beam depending on the type of the end support, for a beam with a simple support at first end and a sliding support at the other

$$
G(x, 0)=0, \quad G^{\prime \prime}(x, 0)=0, G^{\prime}(x, L)=0, G^{\prime \prime \prime}(x, L)=0
$$

(b) continuity conditions of displacement, slope and moment at $\xi=x$, i.e.,

$$
G\left(x, x^{+}\right)=G\left(x, x^{-}\right), G^{\prime}\left(x, x^{+}\right)=G^{\prime}\left(x, x^{-}\right), G^{\prime \prime}\left(x, x^{+}\right)=G^{\prime \prime}\left(x, x^{-}\right)
$$

(c) shear force discontinuity of magnitude one at $\xi=x$, i.e.,

$$
G^{\prime \prime \prime}\left(x, x^{+}\right)-G^{\prime \prime \prime}\left(x, x^{-}\right)=1
$$

Now one can find the unknowns $a-h$ using the eight above-mentioned conditions. Furthermore, it should be pointed out that using conditions given in Eqs. (7), (8) and (9), the coefficients $e-h$ can be expressed in terms of the coefficients $a-d$ as [3]:

$$
e=a-\frac{\cos (\lambda x)}{2 \lambda^{3}}, f=b+\frac{\sin (\lambda x)}{2 \lambda^{3}}, g=c+\frac{\cosh (\lambda x)}{2 \lambda^{3}}, h=d-\frac{\sinh (\lambda x)}{2 \lambda^{3}}
$$

The condition $G(x, 0)=0$ implies $b+d=0$, while $G^{\prime \prime}(x, 0)=0$ gives $-b+d=0$. Thus, $b=d=0$. Recalling that the resonant frequency of the studied beam could be obtained by solving the eigenvalue problem according to Eq. (3) which reveals the free vibration of the system, the frequency parameter $\lambda$ for the case of pinned-sliding Euler-Bernoulli beams satisfy the following equation [9]

$$
\cos (\lambda L)=0
$$

Considering the above relation and Eq. (10), the boundary condition $G^{\prime}(x, L)=0$ yields

$$
c=-\frac{\sinh (\lambda L) \sinh (\lambda x)}{2 \lambda^{3}}-\frac{\cosh (\lambda x)}{2 \lambda^{3}}+\frac{\sinh (\lambda x)}{2 \lambda^{3}}
$$


Similarly, the boundary condition $G^{\prime \prime \prime}(x, L)=0$ gives

$$
c=+\frac{\sinh (\lambda L) \sinh (\lambda x)}{2 \lambda^{3}}-\frac{\cosh (\lambda x)}{2 \lambda^{3}}+\frac{\sinh (\lambda x)}{2 \lambda^{3}}
$$

Obviously, Eqs. (12) and (13) are contradictory. This contradiction is due to the resonant condition. In the next section, it is shown that utilizing the Fredholm Alternative Theorem may be remedial.

\section{Fredholm Alternative Theorem}

The linear boundary-value problem defined by the differential equation

$$
\mathbf{L} u(x)=f(x)
$$

with homogeneous boundary conditions

$$
\mathrm{B}_{i} u(x)=0 \quad i=1,2, \cdots, n
$$

cannot have a unique solution $u(x)$ if the adjoint homogeneous boundary-value problem has a solution $v(x)$ other than $v(x) \equiv 0$. In this case, the given problem (14) can be solved only if $f(x)$ is orthogonal to every $v(x)$, i.e.,

$$
<v, f>=\int_{0}^{L} v(x) f(x) \mathrm{d} x=0
$$

If this condition is satisfied, then the given problem (14) has either no solutions or an infinite number of solutions. Based on the Fredholm Alternative Theorem, the Green's function $G(x, \xi)$ must also satisfy the condition

$$
\mathbf{L} G_{m}(x, \xi)=\delta(x-\xi)+\sum_{k=1}^{n} \alpha_{k} v_{k}(\xi) v_{k}(x)
$$

together with the given boundary conditions (15). Herein, $G_{m}(x, \xi)$ is the modified Green's function and $\alpha$ is a constant. Furthermore, $n$ refers to the number of non-zero solutions of the adjoint homogeneous boundary-value problem.

In our problem, the associated homogeneous equation of Eq. (5) is

$$
\mathbf{L} v=0
$$

or $v^{\prime \prime \prime \prime}-\lambda^{4} v=0$ and the boundary conditions are

$$
\left\{\begin{array}{l}
v(x, 0)=0 \\
v^{\prime \prime}(x, 0)=0 \\
v^{\prime}(x, L)=0 \\
v^{\prime \prime \prime}(x, L)=0
\end{array}\right.
$$


The general solution of Eq. (18) may be written as

$$
v(\xi)=A \sin (\lambda \xi)+B \cos (\lambda \xi)+C \sinh (\lambda \xi)+D \cosh (\lambda \xi)
$$

in which the constants $A-D$ are found by applying the above boundary conditions. By applying the first and second conditions expressed in Eq. (19), one obtains $B=D=0$. Moreover, applying the third and fourth boundary conditions give

$$
C=0
$$

and

$$
\cos (\lambda L)=0
$$

or

$$
\lambda=(2 n-1) \frac{\pi}{2}
$$

Interestingly, Eq. (22) is identical with the frequency equation of the studied beam. Therefore, the non-zero solution of the adjoint homogeneous version of the boundary-value problem, whose existence dictates us to employ the Fredholm Alternative Theorem becomes

$$
v(\xi)=A \sin (\lambda \xi)
$$
gives

Employing the main equation of the Fredholm Alternative Theorem, i.e. Eq. (17)

$$
\mathbf{L}^{*} G_{m}=\delta(x-\xi)+\alpha \cdot \sin (\lambda \xi) \cdot \sin (\lambda x)
$$

The coefficient $\alpha$ should be found by the definition of the adjoint operator as

$$
\int_{0}^{L} v(\xi)\left(\mathbf{L}^{*} G_{m}\right) \mathrm{d} \xi=\int_{0}^{L} G_{m}(\mathbf{L} v) \mathrm{d} \xi
$$

Utilizing Eqs. (18), (24) and (25) results in

$$
\int_{0}^{L} \sin (\lambda \xi)[\delta(x-\xi)+\alpha \cdot \sin (\lambda \xi) \cdot \sin (\lambda x)] \mathrm{d} \xi=0
$$

from which

$$
\alpha=-\frac{2}{L}
$$

Hence, the modified adjoint boundary-value problem takes the form of

$$
G_{m}^{\prime \prime \prime \prime}-\lambda^{4} G_{m}=\delta(x-\xi)+\left(-\frac{2}{L}\right) \sin (\lambda \xi) \cdot \sin (\lambda x)
$$


The solution of Eq. (29) is of the form

$$
G_{m}(x, \xi)=G_{c}(x, \xi)+G_{p}(x, \xi)
$$

where $G_{c}(x, \xi)$ is the complementary solution satisfying the equation

$$
G_{c}^{\prime \prime \prime \prime}-\lambda^{4} G_{c}=\delta(x-\xi)
$$

and $G_{p}(x, \xi)$ is the particular solution corresponding to non-homogeneous term $\left(-\frac{2}{L}\right) \sin (\lambda \xi) \cdot \sin (\lambda x)$ in the right-hand side of Eq. (29).

The corresponding solution of Eq. (31) was previously introduced in Eq. (6). We now consider

$$
G_{p}^{\prime \prime \prime \prime}-\lambda^{4} G_{p}=\left(-\frac{2}{L}\right) \sin (\lambda \xi) \cdot \sin (\lambda x)
$$

The particular solution of Eq. (32) is assumed as $G_{p}=\xi\left[A_{1} \sin (\lambda \xi)+B_{1} \cos (\lambda \xi)\right]$. After substituting this solution and its fourth derivative into Eq. (32) and simplification, one obtains

$$
G_{p}(x, \xi)=-\frac{\sin (\lambda x)}{2 L \lambda^{3}} \xi \cos (\lambda \xi)
$$

Hence, the modified Green's function of the problem is written as

$$
G(x, \xi)=-\frac{\sin (\lambda x)}{2 L \lambda^{3}} \xi \cos (\lambda \xi)+ \begin{cases}a \sin (\lambda \xi)+b \cos (\lambda \xi)+c \sinh (\lambda \xi)+d \cosh (\lambda \xi) & 0 \leq \xi \leq x \\ e \sin (\lambda \xi)+f \cos (\lambda \xi)+g \sinh (\lambda \xi)+h \cosh (\lambda \xi) & x \leq \xi \leq L\end{cases}
$$

subject to the boundary conditions mentioned in Eqs. (7), (8) and (9). In order to find constants $a-h$, the boundary conditions must be imposed, which, for the sake of brevity, we do not present in the process. Moreover, the contradiction is now eliminated and the jump discontinuity condition of the shear is automatically satisfied. After the calculations, one finds the constants as

$$
\begin{gathered}
a(x)=e(x)+\frac{\cos (\lambda x)}{2 \lambda^{3}} \\
b(x)=0 \\
c(x)=\frac{\tanh (\lambda L) \sinh (\lambda x)}{2 \lambda^{3}}-\frac{\cosh (\lambda x)}{2 \lambda^{3}}
\end{gathered}
$$




$$
\begin{gathered}
d(x)=0 \\
f(x)=\frac{\sin (\lambda x)}{2 \lambda^{3}} \\
g(x)=\frac{\tanh (\lambda L) \sinh (\lambda x)}{2 \lambda^{3}} \\
h(x)=-\frac{\sinh (\lambda x)}{2 \lambda^{3}}
\end{gathered}
$$

Thus, one finally obtains the modified Green's function, which in fact, is the dynamic Green's function of the beam studied at the resonant condition, as

$$
\begin{aligned}
G(x, \xi)= & -\frac{\sin (\lambda x)}{2 L \lambda^{3}} \xi \cos (\lambda \xi)+e(x) \sin (\lambda \xi)+\frac{\tanh (\lambda L) \sinh (\lambda x)}{2 \lambda^{3}} \cdot \sinh (\lambda \xi) \\
& +\left\{\begin{array}{lc}
\frac{\cos (\lambda x)}{2 \lambda^{3}} \sin (\lambda \xi)-\frac{\cosh (\lambda x)}{2 \lambda^{3}} \sinh (\lambda \xi) & 0 \leq \xi \leq x \\
\frac{\sin (\lambda x)}{2 \lambda^{3}} \cos (\lambda \xi)-\frac{\sinh (\lambda x)}{2 \lambda^{3}} \cosh (\lambda \xi) & x \leq \xi \leq L
\end{array}\right.
\end{aligned}
$$

where $e(x)$ is an arbitrary constant that depends on $x$ and corresponds to an arbitrary multiple of the Green's function and $\lambda$ is the frequency parameter of the beam which satisfies the Eq. (22).

\section{Discussion and numerical examples}

The primary objective of this section is to develop a formula for the displacement function of the beam and to rigorously investigate the properties of the Green's functions in both cases of absence of resonance and presence of resonance. The displacement function of the beam can be easily obtained by using the definition of the adjoint operator

$$
\int_{0}^{L} G_{m}\left(u^{\prime \prime \prime \prime}-\lambda^{4} u\right) \mathrm{d} \xi=\int_{0}^{L} u\left(G_{m}^{\prime \prime \prime \prime}-\lambda^{4} G_{m}\right) \mathrm{d} \xi
$$

or

$$
\int_{0}^{L} G_{m} \phi(\xi) \mathrm{d} \xi=\int_{0}^{L} u(\xi) \delta(\xi-x) \mathrm{d} \xi-\int_{0}^{L} u(\xi)\left[\left(\frac{2}{L}\right) \sin (\lambda x) \sin (\lambda \xi)\right] \mathrm{d} \xi
$$


Utilizing the fundamental property of the Dirac function yields

$$
u(x)=\int_{0}^{L} G_{m}(x ; \xi) \phi(\xi) \mathrm{d} \xi+\left(\frac{2}{L}\right) \sin (\lambda x) \int_{0}^{L} u(\xi) \sin (\lambda \xi) \mathrm{d} \xi
$$

Three points are of interest: (1) As a verification purpose, the displacement function must satisfy the four boundary conditions at both ends of the beam. Therefore

$$
\begin{aligned}
& u(0)=E(0) \int_{0}^{L} \sin (\lambda \xi) \phi(\xi) \mathrm{d} \xi=0 \\
& u^{\prime \prime}(0)=E^{\prime \prime}(0) \int_{0}^{L} \sin (\lambda \xi) \phi(\xi) \mathrm{d} \xi=0 \\
& u^{\prime}(L)=\left[\frac{\sin (\lambda L)}{2 \lambda^{2}}-E^{\prime}(L)\right] \int_{0}^{L} \sin (\lambda \xi) \phi(\xi) \mathrm{d} \xi=0 \\
& u^{\prime \prime \prime}(L)=\left[\frac{\sin (\lambda L)}{2}+E^{\prime \prime \prime}(L)\right] \int_{0}^{L} \sin (\lambda \xi) \phi(\xi) \mathrm{d} \xi=0
\end{aligned}
$$

It is seen that the above equations hold only if

$$
\int_{0}^{L} \sin (\lambda \xi) \phi(\xi) \mathrm{d} \xi=0
$$

which states the required condition in Eq. (16). (2) The unknown coefficient $e(x)$ does not affect the displacement of the beam. This happens because, taking into consideration Eq. (40), the forcing function $\phi(x)$ must be orthogonal to the non-zero solution of the homogeneous equation (18) and thus the constant vanishes; (3) It can be easily shown the integral part in the last term of the displacement function $u(x)$ has a constant amount i.e.,

$$
\int_{0}^{L} u(\xi) \sin (\lambda \xi) \mathrm{d} \xi=k
$$

where $k$ is a constant. Remarkably, this constant is arbitrary and gives rise to an infinite number of displacement function.

The static Green's function, i.e., the Green's function for near zero frequency $\lambda=0.0001 \frac{\pi}{L}$ is plotted in Figure 2 [4]. It is interesting to point out that the Green's function of the problem is symmetric about line $x=\xi$. One can also use Table 1 which presents of the amounts of the static Green's function at different points. Interestingly, the symmetry of the given values is observed. 


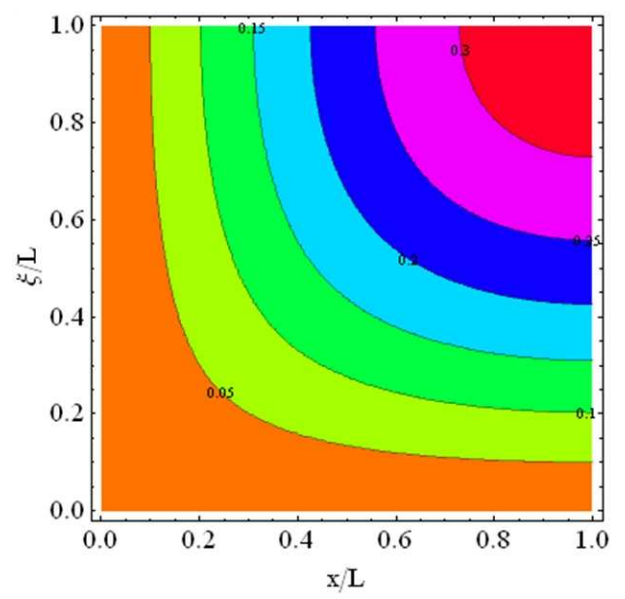

Fig. 2. Contour of the Green's function for $\lambda=0.0001 \pi / 2 L$

Figure 3 shows the contours of the Green's function of the beam in the absence of resonance at frequencies approaching the first natural frequency of the beam and the Green's function of the beam at the first natural frequency of the beam which corresponds to the resonant condition. The function $e(x)$ in the case related to the resonance, for the sake of verification, is selected as

$$
e(x)=\frac{\tan (0.9999 \lambda L) \sin (\lambda x)}{2 \lambda^{3}}
$$

where $\lambda=\pi / 2 L$. Figure 3a depicts the Green's function of the beam in the absence of resonance at frequency $\lambda=0.9999 \pi / 2 L$ while Figure $3 \mathrm{~b}$ shows the Green's function of the beam in the presence of resonance at frequency $\lambda=\pi / 2 L$. Besides, Figure 3 represents the three-dimensional plot of the Green's function in the absence of resonance for Figure 3 represents the three-dimensional plot of the Green's function in the absence of resonance for $\lambda=0.9999 \pi / 2 L, \lambda=\pi / L, \lambda=2.9999 \pi / 2 L$ and $\lambda=2 \pi / L$. In addition, Tables $2-4$ demonstrate the values of the Green's function at different locations and different amounts of $\lambda: \lambda=0.9999 \pi / 2 L, \lambda=\pi / 2 L$ and $\lambda=\pi / L$.

\section{Conclusion}

The dynamic analysis of an Euler-Bernoulli beam at resonance is studied. The beam studied has a simple support as well as a sliding support. The Green's function method is utilized in order to perform the analysis. The governing differential equation of motion of the Euler-Bernoulli beams is found using the well-known 


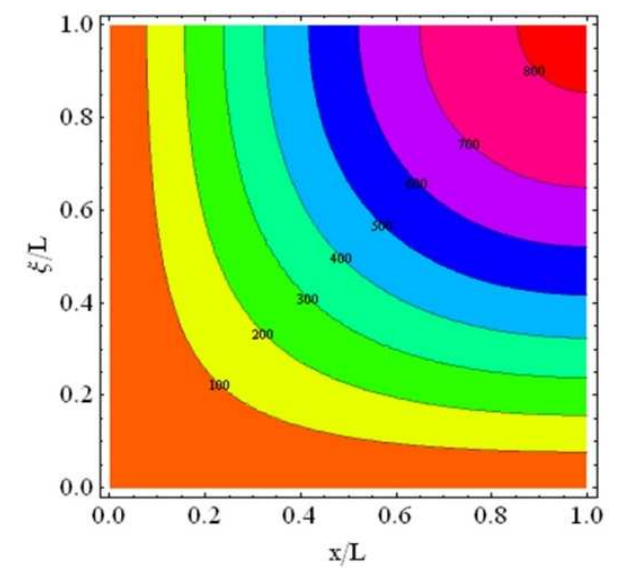

(a)

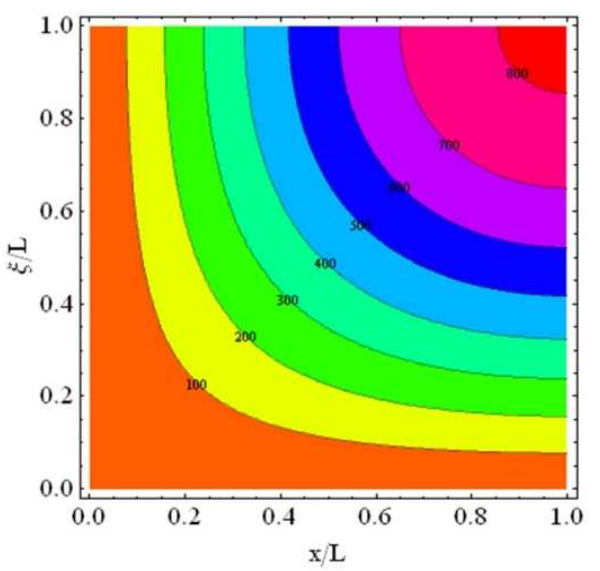

(b)

Fig. 3. Contours of the Green's function (a) $\lambda=0.9999 \pi / 2 L$ (b) $\lambda=\pi / 2 L$

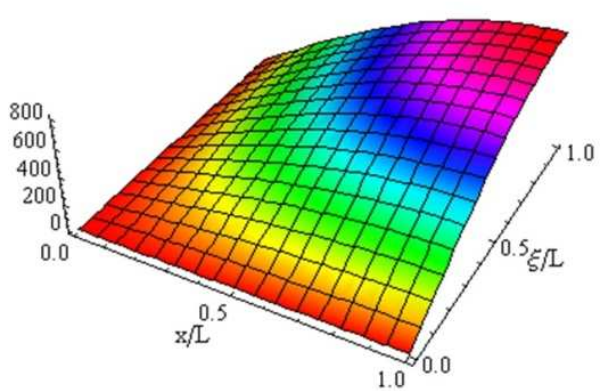

(a)

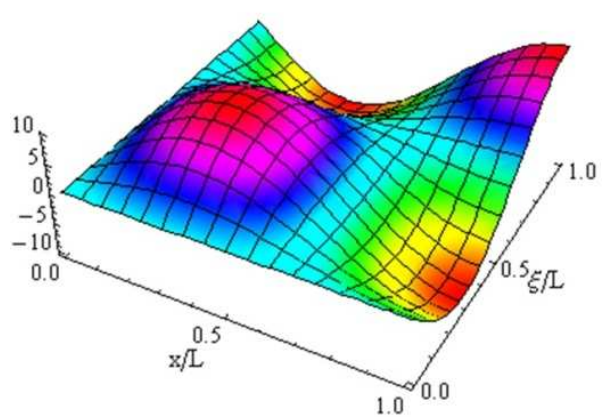

(c)

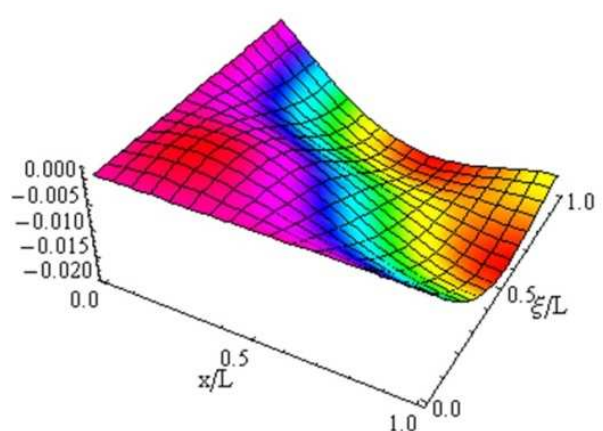

(b)

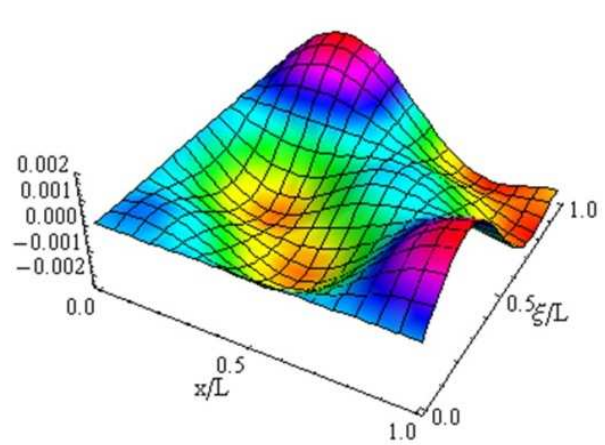

(d)

Fig. 4. Green's functions for different amounts of $\lambda$ (a) $\lambda=0.9999 \pi / 2 L$ (b) $\lambda=\pi / L$ (c) $\lambda=0.9999 \pi / 2 L$ (d) $\lambda=2 \pi / L$ 
Table 1

The values of the static Green's function

\begin{tabular}{|c|c|c|c|c|c|c|c|c|c|c|c|c|}
\hline \multirow{2}{*}{\multicolumn{2}{|c|}{$\mathrm{G}(\mathrm{x} ; \xi)$}} & \multicolumn{11}{|c|}{$\mathrm{x} / \mathrm{L}$} \\
\hline & & 0 & 0.1 & 0.2 & 0.3 & 0.4 & 0.5 & 0.6 & 0.7 & 0.8 & 0.9 & 1 \\
\hline \multirow{11}{*}{ ज्ञ } & 0 & 0 & 0 & 0 & 0 & 0 & 0 & 0 & 0 & 0 & 0 & 0 \\
\hline & 0.1 & 0 & 0.0093 & 0.0178 & 0.0253 & 0.0318 & 0.0373 & 0.0418 & 0.0453 & 0.0478 & 0.0493 & 0.0498 \\
\hline & 0.2 & 0 & 0.0178 & 0.0347 & 0.0497 & 0.0627 & 0.0737 & 0.0827 & 0.0897 & 0.0947 & 0.0977 & 0.0987 \\
\hline & 0.3 & 0 & 0.0253 & 0.0497 & 0.0720 & 0.0915 & 0.1080 & 0.1215 & 0.1320 & 0.1395 & 0.1440 & 0.1455 \\
\hline & 0.4 & 0 & 0.0318 & 0.0627 & 0.0915 & 0.1173 & 0.1393 & 0.1573 & 0.1713 & 0.1813 & 0.1873 & 0.1893 \\
\hline & 0.5 & 0 & 0.0373 & 0.0737 & 0.1080 & 0.1393 & 0.1667 & 0.1892 & 0.2067 & 0.2192 & 0.2267 & 0.2292 \\
\hline & 0.6 & 0 & 0.0418 & 0.0827 & 0.1215 & 0.1573 & 0.1892 & 0.2160 & 0.2370 & 0.2520 & 0.2610 & 0.264 \\
\hline & 0.7 & 0 & 0.0453 & 0.0897 & 0.1320 & 0.1713 & 0.2067 & 0.2370 & 0.2613 & 0.2788 & 0.2893 & 0.2928 \\
\hline & 0.8 & 0 & 0.0478 & 0.0947 & 0.1395 & 0.1813 & 0.2192 & 0.2520 & 0.2788 & 0.2987 & 0.3107 & 0.3147 \\
\hline & 0.9 & 0 & 0.0493 & 0.0977 & 0.1440 & 0.1873 & 0.2267 & 0.2610 & 0.2893 & 0.3107 & 0.3240 & 0.3285 \\
\hline & 1 & 0 & 0.04983 & 0.0987 & 0.1455 & 0.1893 & 0.22917 & 0.264 & 0.29283 & 0.3147 & 0.3285 & 0.3333 \\
\hline
\end{tabular}

Table 2

The values of the Green's function for $\lambda=0.9999 \pi / 2 L$

\begin{tabular}{|c|c|c|c|c|c|c|c|c|c|c|c|c|}
\hline \multirow{2}{*}{\multicolumn{2}{|c|}{$\mathrm{G}(\mathrm{x} ; \xi)$}} & \multicolumn{11}{|c|}{$x / L$} \\
\hline & & 0 & 0.1 & 0.2 & 0.3 & 0.4 & 0.5 & 0.6 & 0.7 & 0.8 & 0.9 & 1 \\
\hline \multirow{11}{*}{ is } & 0 & 0 & 0 & 0 & 0 & 0 & 0 & 0 & 0 & 0 & 0 & 0 \\
\hline & 0.1 & 0 & 20.1024 & 39.7093 & 58.3378 & 75.5294 & 90.8611 & 103.9550 & 114.4900 & 122.2050 & 126.9120 & 128.4940 \\
\hline & 0.2 & 0 & 39.7093 & 78.4402 & 115.2390 & 149.1990 & 179.4850 & 205.3510 & 226.1610 & 241.4020 & 250.6990 & 253.8240 \\
\hline & 0.3 & 0 & 58.3378 & 115.2390 & 169.3010 & 219.1940 & 263.6890 & 301.6900 & 332.2630 & 354.6550 & 368.3140 & 372.9050 \\
\hline & 0.4 & 0 & 75.5294 & 149.1990 & 219.1940 & 283.7910 & 341.4000 & 390.6010 & 430.1840 & 459.1750 & 476.8600 & 482.8040 \\
\hline & 0.5 & 0 & 90.8611 & 179.4850 & 263.6890 & 341.4000 & 410.7030 & 469.8940 & 517.5130 & 552.3900 & 573.6650 & 580.8160 \\
\hline & 0.6 & 0 & 103.9550 & 205.3510 & 301.6900 & 390.6010 & 469.8940 & 537.6150 & 592.0990 & 632.0030 & 656.3450 & 664.5260 \\
\hline & 0.7 & 0 & 114.4900 & 226.1610 & 332.2630 & 430.1840 & 517.5130 & 592.0990 & 652.1050 & 696.0540 & 722.8640 & 731.8740 \\
\hline & 0.8 & 0 & 122.2050 & 241.4020 & 354.6550 & 459.1750 & 552.3900 & 632.0030 & 696.0540 & 742.9660 & 771.5840 & 781.2020 \\
\hline & 0.9 & 0 & 126.9120 & 250.6990 & 368.3140 & 476.8600 & 573.6650 & 656.3450 & 722.8640 & 771.5840 & 801.3040 & 811.2930 \\
\hline & 1 & 0 & 128.4940 & 253.8240 & 372.9050 & 482.8040 & 580.8160 & 664.5260 & 731.8740 & 781.2020 & 811.2930 & 821.4070 \\
\hline
\end{tabular}

Table 3

The values of the Green's function for $\lambda=\pi / 2 L$

\begin{tabular}{|c|c|c|c|c|c|c|c|c|c|c|c|c|}
\hline \multirow{2}{*}{\multicolumn{2}{|c|}{$\mathrm{G}(\mathrm{x} ; \xi)$}} & \multicolumn{11}{|c|}{$x / L$} \\
\hline & & 0.0 & 0.1 & 0.2 & 0.3 & 0.4 & 0.5 & 0.6 & 0.7 & 0.8 & 0.9 & 1.0 \\
\hline \multirow{11}{*}{ Eis } & 0.0 & 0 & 0 & 0 & 0 & 0 & 0 & 0 & 0 & 0 & 0 & 0 \\
\hline & 0.1 & 0 & 20.0984 & 39.7012 & $\mathbf{5 8 . 3 2 5 7}$ & 75.5133 & 90.8410 & 103.9310 & 114.4620 & 122.1740 & 126.8770 & 128.4550 \\
\hline & 0.2 & 0 & 39.7013 & 78.4243 & 115.2150 & 149.1670 & 179.4450 & 205.3030 & 226.1060 & 241.3390 & 250.6300 & 253.7480 \\
\hline & 0.3 & 0 & 58.3261 & 115.2150 & 169.2660 & 219.1470 & 263.6300 & 301.6200 & 332.1820 & 354.5630 & 368.2120 & 372.7930 \\
\hline & 0.4 & 0 & 75.5143 & 149.1690 & 219.1490 & 283.7310 & 341.3240 & 390.5100 & 430.0790 & 459.0560 & 476.7280 & 482.6590 \\
\hline & 0.5 & 0 & 90.8428 & 179.4480 & 263.6340 & 341.3270 & 410.6120 & 469.7850 & $\mathbf{5 1 7 . 3 8 7 0}$ & 552.2470 & 573.5060 & 580.6410 \\
\hline & 0.6 & 0 & 103.9340 & 205.3090 & 301.6280 & 390.5170 & 469.7890 & 537.4910 & 591.9550 & 631.8390 & 656.1630 & 664.3270 \\
\hline & 0.7 & 0 & 114.4670 & 226.1150 & 332.1940 & 430.0920 & 517.3980 & 591.9620 & 651.9460 & 695.8740 & 722.6630 & 731.6550 \\
\hline & 0.8 & 0 & 122.1810 & 241.3530 & 354.5810 & 459.0770 & 552.2670 & 631.8570 & 695.8850 & 742.7740 & 771.3700 & 780.9670 \\
\hline & 0.9 & 0 & 126.8870 & 250.6480 & 368.2380 & 476.7580 & 573.5380 & 656.1930 & 722.6880 & 771.3840 & 801.0820 & 811.0500 \\
\hline & 1.0 & 0 & 128.4680 & 253.7730 & 372.8280 & 482.7010 & 580.6870 & 664.3720 & 731.6960 & 780.9990 & 811.0680 & 821.1600 \\
\hline
\end{tabular}

The values of the Green's function for $\lambda=\pi / L$

Table 4

The values of the Green's function for $\lambda=\pi / L$

\begin{tabular}{|c|c|c|c|c|c|c|c|c|c|c|c|c|}
\hline \multirow{2}{*}{\multicolumn{2}{|c|}{$G(x ; \xi)$}} & \multicolumn{11}{|c|}{$\mathrm{x} / \mathrm{L}$} \\
\hline & & 0 & 0.1 & 0.2 & 0.3 & 0.4 & 0.5 & 0.6 & 0.7 & 0.8 & 0.9 & 1 \\
\hline \multirow{11}{*}{ ज्ञ } & 0 & 0 & 0 & 0 & 0 & 0 & 0 & 0 & 0 & 0 & 0 & 0 \\
\hline & 0.1 & 0 & 0.0010 & 0.0013 & 0.0009 & 0.0000 & -0.0011 & -0.0024 & -0.0036 & -0.0046 & -0.0052 & -0.0054 \\
\hline & 0.2 & 0 & 0.0013 & 0.0019 & 0.0013 & -0.0002 & -0.0023 & -0.0047 & -0.0069 & -0.0088 & -0.0100 & -0.0104 \\
\hline & 0.3 & 0 & 0.0009 & 0.0013 & 0.0008 & -0.0011 & -0.0038 & -0.0069 & -0.0099 & -0.0124 & -0.0140 & -0.0146 \\
\hline & 0.4 & 0 & 0.0000 & -0.0002 & -0.0011 & -0.0028 & -0.0056 & -0.0090 & -0.0123 & -0.0151 & -0.0169 & -0.0176 \\
\hline & 0.5 & 0 & -0.0011 & -0.0023 & -0.0038 & -0.0056 & -0.0080 & -0.0111 & -0.0142 & -0.0169 & -0.0187 & -0.0193 \\
\hline & 0.6 & 0 & -0.0024 & -0.0047 & -0.0069 & -0.0090 & -0.0111 & -0.0132 & -0.0156 & -0.0178 & -0.0193 & -0.0198 \\
\hline & 0.7 & 0 & -0.0036 & -0.0069 & -0.0099 & -0.0123 & -0.0142 & -0.0156 & -0.0168 & -0.0180 & -0.0189 & -0.0192 \\
\hline & 0.8 & 0 & -0.0046 & -0.0088 & -0.0124 & -0.0151 & -0.0169 & -0.0178 & -0.0180 & -0.0179 & -0.0180 & -0.0180 \\
\hline & 0.9 & 0 & -0.0052 & -0.0100 & -0.0140 & -0.0169 & -0.0187 & -0.0193 & -0.0189 & -0.0180 & -0.0170 & -0.0167 \\
\hline & 1 & 0 & -0.0054 & -0.0104 & -0.0146 & -0.0176 & -0.0193 & -0.0198 & -0.0192 & -0.0180 & -0.0167 & -0.0161 \\
\hline
\end{tabular}


Fourier transform. Then, the Green's function of the problem is derived in which a contradiction occurs. To remedy this problem, the Fredholm Alternative Theorem is employed. This theorem imposes a requirement so that the problem will have a solution. If this requirement is satisfied, a particular term for the Green's function is introduced. This new particular term eliminates the mentioned contradiction and consequently, the modified Green's function is available, which is not symmetric in general. Some tables and graphs are also presented.

\section{References}

[1] Xu M., Cheng D., A new approach to solving a type of vibration problem, J. Sound Vibrat. 1994, 177, 565-571.

[2] Mohamad A.S., Tables of Green's function for the theory of beam vibrations with general intermediate appendages, Int. J. Solids Structures 1994, 31, 93-102.

[3] Kukla S., Application of Green's functions in frequency analysis of Timoshenko beams with oscillators, J. Sound Vibrat. 1997, 205, 355-393.

[4] Foda M.A., Abduljabbar Z., A dynamic Green function formulation for the response of a beam structure to a moving mass, J. Sound Vibrat. 1998, 210, 295-306.

[5] Abu-Hilal M., Forced vibration of Euler-Bernoulli beams by means of dynamic Green function, J. Sound Vibrat. 2003, 267, 191-207.

[6] Failla G., Santini A., On Euler-Bernoulli discontinuous beam solutions via uniform-beam Green's function, Int. J. Solids Structures 2007, 44, 7666-7687.

[7] Failla G., Closed-form solutions for Euler-Bernoulli arbitrary discontinuous beams, Arch. Appl. Mech. 2011, 81, 605-628.

[8] Azizi A., Saadatpoor M., Mahzoon M., Using spectral element method for analyzing continuous beams and bridges subjected to a moving load, Appl. Math. Model 2012, 36, 3580-3592.

[9] Rao S.S., Vibration of Continuous Systems, John Wiley and Sons, U.S. 2007.

[10] Korn G.A., Korn T.M., Mathematical Handbook for Scientists and Engineers, second ed., McGraw-Hill, U.S. 1968. 